\title{
On the long run sustainability of small jurisdictions*
}

\author{
Skerdilajda Zanaj ${ }^{\dagger}$ Patrice Pieretti, Benteng Zou. \\ CREA, UNIVERSITY OF LUXEMBOURG
}

December 2, 2019

\begin{abstract}
In this paper, we analyse the long run sustainability of a small jurisdiction open to capital flows. Policy instruments used to reach an efficient steady state (when possible) are capital taxes and attractive public infrastructures. We first show that there exists one long run efficient equilibrium which is saddle-point stable. If capital mobility is high enough, the small economy is always able to converge to a long run efficient state by a one-shot adjustment of the share of taxes allocated to infrastructure. If capital mobility is too low, convergence to long run efficiency may be impossible. In this case, an eventual economic collapse can occur.
\end{abstract}

Keywords: Economic dynamics, foreign direct investments, spatial dynamic competition.

JEL classification: H25, H73, F13, F15, O30, O43.

${ }^{*}$ We gratefully acknowledge discussions with Raouf Boucekkine, Herbert Dawid, Pierre Picard, Ornella Tarola and Myrna Wooders. We thank the editor and two anonymous referees for very useful comments. The usual disclaimer applies.

${ }^{\dagger}$ Corresponding author: 162a, Avenue de la Faïencerie, L-1511 Luxembourg, Luxembourg. E-mail: skerdilajda.zanaj@uni.lu 


\section{Introduction}

It is well-known that small economies suffer from very limited capital and labor resources both in amount and in variety. Their small home market size prevents them from exploiting scale and scope economies. It is therefore not surprising that small states need to be open to international trade and capital flows (Alesina and Wacziarg, 1998). Because domestic capital is relatively scarce in small economies, attracting foreign investments is an important way to fill this gap. Indeed, it has been well documented that small states or jurisdictions tend to get more private foreign capital as a ratio of total capital formation (Streeten, 1993). As shown in Han et al (2014), the ratio of FDI flows to the gross fixed capital formation is much higher in small countries (i.e., population less than two million) than in large countries (i.e., population in excess of 30 million). ${ }^{1}$

In addition, small countries are also more vulnerable than other countries to climate variability and natural disasters (e.g., Briguglio, 1995). Accordingly, openness to trade and foreign investment must come along a path of sustainable development which is key for their long run survival. Sustainable development for small countries in this paper is intended as "development which meets the needs of the present without compromising the ability of future generations to meet their own needs" from the "Brundtland Report" (1987) (see for instance Holden et al 2014 and White (2013)). Indeed, the focus of this paper is to analyze the long run economic sustainability of a small country in a globalized world.

In recent years, attracting foreign capital to invest in eco business has become a trend. For instance, it is documented that more 200 eco businesses are located in Luxembourg mainly operating in the fields of renewable energy, waste management, water and environmentally sound construction. In order to support these companies in their efforts, the government offers financial support, and it also helps establishing networks of expertise to guarantee ultimately their country sustainability. Over $99 \%$ of electricity production and almost $80 \%$ of total energy production in Iceland comes from hydropower and geothermal power making meeting buildings quite naturally eco-friendly. There are many other examples: the growing use of wind farms, the introduction of energy performance certificates in the building sector as well as the efforts implemented for more electric public transport. The Icelandic government has stated an objective of making Iceland the first nation to use only renewable energy for its power in the near future according to the Icelandic National Strategy for sustainable development.

Our purpose is to explore the optimal policy mix in terms of public investment and taxation for

\footnotetext{
${ }^{1}$ Moreover, capital inflows may also be a critical contributor to the growth and development of small jurisductions (Read, 2008). Some empirical evidence shows a positive impact of FDI on economic growth and the possibility of spillover effects to local firms (Castellani and Zanfei, 2006).
} 
the long run feasibility of a small jurisdictions. In this paper, the term jurisdiction has to be used in a general sense. It can be a country, a local government within a federation, a region or a city having enough power to tax and to decide on local infrastructure. ${ }^{2}$ Thus, we assume that the policy-makers of the small jurisdiction use a mix of two instruments in a dynamic optimization framework to promote the durability of their economy. More exactly, the policy-mix consists in attracting foreign capital through low taxes and/or high level of public goods, which enhance firms' productivity. Public goods can cover a wide range of infrastructures, services and environmental regulations provided by the local government that are attractive to firms by increasing their productivity. These public goods can be intended as infrastructure needed for adaptation to climate change or mitigation of climate change. Think, for instance, of alert mechanisms to natural disasters, or projects like artificial barriers (e.g., the MOSE in Venice); plants for the treatment of waters (desalination). Consequently, capital locates according to differentials in offered public good levels and tax differentials. Hence, a jurisdiction may not reduce its attractiveness by a unilateral increase in taxes if foreign investors are compensated by more infrastructure provision. Foreign investors are ready to pay higher taxes because infrastructure become more valuable to them (e.g., Haufler, 1998, Pieretti and Zanaj, 2011).

In this research, the small jurisdiction is small enough to consider the rest of the world's choices as exogenously given. This does not mean that small jurisdictions cannot grow in terms of productive capacity by attracting a high volume of foreign direct investments even if its population size remains exiguous. What we actually assume is that their ability to grow bigger than large jurisdiction is limited. ${ }^{3}$ For example, if smallness does not only rely on population size but also on a territorial criterion, the existence of limited usable land can be considered as an absorptive constraint for foreign investments. ${ }^{4}$ Another limitation can result from the smallness of the native population since it constraints the availability of administrative and public resources which are necessary to increase infrastructure provision. Finally, a high ratio of foreign-owned firms may be perceived as a loss of economic independence and induce some resistance to new FDIs.

The literature has investigated the role of jurisdictions' size on their capacity to attract capital. Recent papers show that small economies can be attractive not only for tax reasons but also for their provision of public infrastructures (Justman et al., 2005, Zissimos and Wooders, 2008, Hindriks et al., 2008, Pieretti and Zanaj, 2011). This paper extends this literature by modelling the dynamics of a small economy's strategies to attract foreign investments. More precisely, we study a small jurisdic-

\footnotetext{
${ }^{2}$ During the past decades there has been an increasing demand for fiscal decentralization of governments. This trend results in giving more power to tax and to decide on public expenditures to sub-national entities.

${ }^{3}$ Exceptions could however exist if we focus for example one particular sector like banking and finance.

${ }^{4}$ According to Armstrong and Read (1995), the small size constitutes a significant impediment to sustained economic growth, especially in the case of island and land-locked states.
} 
tion's dynamic choice of taxes that are used to afford public goods that enhance firms' productivity. Applying the Pontryagin's maximum principle (see, for example, Boucekkine et al. (2007)), we characterize the potential steady states attainable by the small economy.

The dynamic interactions among jurisdictions to attract mobile factors have already been analyzed within a repeated game framework. The main issue studied by this literature is the tax coordination problem between symmetric regions (Cardarella et al., 2002, Catenaro and Vidal, 2006, Itaya et al., 2008). However, the purpose of this paper is not to model a game between jurisdictions. We rather focus on the long run decisions of a small open economy facing exogenously given tax and infrastructure choices of the rest of the world. The world is thus divided into two unequal sized regions where size refers to the magnitude of the population, which coincides with the number of capital-owners who are simultaneously entrepreneurs and workers. In our paper, we consider that only the economic size of jurisdictions can vary endogenously as a consequence of public policy, but that their territorial borders remain unchanged. Finally, our paper is related to literature on (uneven) emissions taxation of polluting firms and their corresponding relocation choices (for instance, Exbrayat et al 2016; Sanna-Randaccio and Sestini, 2012, Sanna-Randaccio, Sestini and Tarola 2017). In particular, Sanna-Randaccio, Sestini and Tarola (2017) tackle the so-called "reverse market asymmetry" scenario, i.e. when the small country enacts a tougher environmental policy as compared with the rest of the world, it becomes extremely likely that firms relocate abroad their productive activities. This scenario could be one of the drivers of the long run economic collapse examined in the present paper. In order to avoid such a collapse, the small jurisdiction may resort to a policy mix allowing for R\&D investment subsides, public R\&D investment or other public infrastructure. In other words, relying on these public infrastructure may represent a powerful centripetal force, leading the small country to economic survival.

The results of the paper can be summarized as follows. First, we demonstrate that there exists one long run efficient steady state that guarantees the long run existence of the small jurisdiction. Then, we address the question of convergence to this efficient state. When capital mobility is high enough, we demonstrate that the small economy can ensure convergence toward long run efficiency by allocating an adequate share of taxes to infrastructure expenditures. This share can be chosen in a non empty set of possible values. In case of perfect capital mobility we show, as a corollary, that the efficient steady state is always attainable. Consequently, the economic fate of small jurisdictions is in their own hands when capital mobility is not too low. Then paradoxically, there is room for efficient economic policy even if small jurisdictions are highly depending on forces outside their control. When capital mobility is too low, there may exist no way to converge to long run efficiency and economic collapse can be a possible end-state. Along this divergence path, the small economy 
would vainly try to retain capital by tax dumping and thus drive its infrastructure expenditures to zero. Nevertheless, this scenario does not occur if the public benefit of infrastructures net of taxes provided by the rest of the world is negative.

The paper is organized as follows. The next section develops a dynamic model and the optimal conditions by applying the Pontryagin's Maximum Principle. Section 3 derives and analyses the steady states of the model. Finally, section 4 concludes.

\section{The model}

The world is composed of two regions of unequal population size. ${ }^{5}$ We consider the smaller region as the home economy and the larger one as the rest of the world, indifferently. We also assume that the members of both jurisdictions are at the same time entrepreneurs and workers and each of them owns one unit of productive capital. Consequently, in each jurisdiction the endowment in human resources and physical capital grows in proportion to the human population. Moreover, size is equivalent to the number of firms located in its territory. It follows that the economic size of each jurisdiction can change over time as a result of international relocations of firms. However, the political size, characterized by the geographical area and the native population, will remain unchanged through time. ${ }^{6}$

At time $t=0$, these jurisdictions are represented on an interval $\left[-S(0), S^{*}(0)\right] .^{7}$ The size of the small one is $S(0)$ and extends from $-S(0)$ to 0 which corresponds to the "border". The rest of the world has a size of $S^{*}(0)$ with $S(0)<S^{*}(0)$ and extends from 0 to $S^{*}(0)$. The firm owners in both jurisdictions are evenly distributed on their respective sub-interval according to their propensity to invest outside their home location. As in Ogura (2006), we assume that the population of investors is heterogeneous in the degree of their attachment to home. ${ }^{8}$

The spatial setting we use is intended to characterize firms' location preferences. More exactly, we assume that firms located closer to the extremities of $\left[-S(0), S^{*}(0)\right]$ are the most attached to their current location. Conversely, the closer firms are to the "border" 0 , the less they are attached to their

\footnotetext{
${ }^{5}$ Country size may be defined by its population, by its area, or by its national income (Streeten, 1993). In our paper, we focus on the population aspect rather than on the spatial size.

${ }^{6}$ For the sake of simplicity, and in line with the purpose of highlighting the role of public infrastructure, we assume that there is only one sector in each country. A paper that relaxes such an assumption assuming three sectors is $\mathrm{Hu}$ and Mino (2014) who analyze how capital accumulation affects the process of structural transformation of a small open economy.

${ }^{7}$ The superscript" * "refers to the large (foreign) jurisdiction.

${ }^{8}$ Heterogeneity in home attachment was first considered in the fiscal competition literature by Mansoorian and Myers (1993).
} 
territory and the easier they are able to relocate abroad. This means that a firm of type $h \in[-S(0), 0]$ located in the home country incurs a disutility of relocating abroad which equals $k \cdot d$, where $d$ is the "distance" between $h$ and $0 .{ }^{9}$ The coefficient $k$ represents the unit cost of moving capital abroad which can also be interpreted as the degree of international integration.

Now assume that each population member of both jurisdictions owns one unit of capital which she combines with her labor to set up a firm to produce $q+a_{i}, a_{i}=a, a^{*}$, units of a final good, where $q$ is the private component of (gross) productivity. The fraction $a_{i}$ of the produced good depends on the public input supplied by the home (foreign) jurisdiction. ${ }^{10}$ This makes the jurisdiction more attractive to foreign financial firms and increases the attachment to home of domestic financial firms, ceteris paribus. The produced output is sold in a competitive (world) market at a given price normalized to one. Assuming that both countries have equal access to a common market this assumption implies that the small jurisdiction does not suffer from a reduced home market. We further suppose that the unit production cost is constant and equal to zero without loss of generality.

We now adopt a temporal perspective of the above setting. Each period $t \in[\Delta t,+\infty)$, (for any $\Delta t>0$ ) (federal or local) governments update their choice in terms of offered public goods and taxes. ${ }^{11}$ We assume that the total number of entrepreneurs, $S(t)+S^{*}(t)$ will be constant over time $t$ and normalized to one. Since firms may move, the relative size of both jurisdictions will change with $t$. In the following, we focus on the behavior of the small country whose economic size can change over time. However, as explained above, the maximum amount of FDIs will be limited so that the economic size of the home jurisdiction always remains small relative to the rest of the world. Accordingly, $S(t)$ is small enough to consider the rest of the world's choices as exogenously given. In order to ensure the consistency of this assumption, the following state-space constraint appears in the dynamic optimization problem.

$$
0 \leq S(t)<\bar{S}<\frac{1}{2} \text { for } t \in[0,+\infty) .
$$

Let $T(t)$ and $T^{*}(t)$ be the source-based tax rates levied on capital in the small and the large jurisdictions respectively. Accordingly, the tax revenues equal respectively $S(t) \cdot T(t)$ and $S^{*}(t) T^{*}(t)$. We

\footnotetext{
${ }^{9}$ In the large country, the attachment to home of a firm of type $h^{*} \in\left[0, S^{*}(0)\right]$ is defined by the distance $d\left(0, h^{*}\right)>0$.

${ }^{10}$ The public input satisfies the local public good characteristic, which means that it is jointly used without rivalry by firms located in the same jurisdiction. It follows that the benefits and the costs of these good only accrue at the jurisdictional level. As in Zissimoss and Wooders (2008), we shall abstract from congestion costs . Taking account of congestion would complicate our framework without improving qualitatively the results. Moreover, if the public input represents immaterial goods as law and regulations (protecting intellectual property, specifying accurate dispute resolution rules,...), the absence of congestion is easily justified by the particular nature of these goods.

${ }^{11}$ Notice that we assume there are no sunk cost on the investment or that our unit of time $t$ is long enough to cancel the sunk cost of investment.
} 
assume one unit of tax revenue can be transformed into one unit of infrastructure and we denote by $\zeta_{i}\left(0<\zeta_{i}<1, \zeta_{i}=\zeta, \zeta^{*}\right)$ the share of tax revenue allocated to the infrastructure expenditures in jurisdiction $i$. The coefficient $\zeta$ can also be interpreted as the ability to transform taxes into infrastructures expenditures. This ability can be a technical feature of funding public expenditures but it can also reflect priorities emanating from collective decisions.

Supposing that the public good depreciates at a rate $\delta>0$, the law of motion for the infrastructure stock are given by

$$
\begin{aligned}
\dot{a}(t) & =-\delta a(t)+\zeta S(t) T(t), \\
\dot{a}^{*}(t) & =-\delta a^{*}(t)+\zeta^{*} S^{*}(t) T^{*}(t) .
\end{aligned}
$$

Assume now that an entrepreneur of type $h(t)$, initially located at home, considers to stay at home or to invest her/his physical capital abroad. If she/he decides not to move, her/his profit is given by $^{12}$

$$
\pi(t)=q(t)+a(t)-T(t)
$$

If she invests abroad, her/his profit becomes

$$
\pi^{*}(t)=q(t)+a^{*}(t)-T^{*}(t)-k \cdot d(t)
$$

Furthermore, consider that this capital-owner is indifferent between investing abroad and staying at home. Then it follows that

$$
q(t)+a(t)-T(t)=q(t)+a^{*}(t)-T^{*}(t)-k \cdot d(t) .
$$

After setting $b^{*}(t)=\frac{a^{*}(t)-T^{*}(t)}{k}$, we obtain

$$
x\left(t, a, a^{*}, T, T^{*}\right)=b^{*}(t)-\frac{a(t)-T(t)}{k},
$$

with $x(t)=d(t)>0$ if the rest of the world attracts capital from the small jurisdiction; and $x(t)=$ $-d(t)<0$ if capital moves in the opposite direction. In other words, the small jurisdiction attracts capital if the net gain of investing abroad, i.e. $a^{*}(t)-T^{*}(t)$, is smaller than the net gain of staying at home, $a(t)-T(t)$, after taking into account the total mobility cost $k x$. In the sequel, we impose that $a^{*}(t)-T^{*}(t) \neq 0$.

The motion equation of the size variable $S(t)$ of the small economy is then given by,

$$
\dot{S}(t)=-x=\frac{a(t)-T(t)}{k}-b^{*}(t)
$$

\footnotetext{
${ }^{12}$ For sake of simplicity, we assume that $q$ is such that the profit of each firm is positive for all equilibrium level of public goods and taxes.
} 
with the initial condition, $\frac{1}{2}>\bar{S}>S(0)>0$.

Note that the relocation of a subset of firms at each period alters the ranking of firms' attachment to home. Therefore, we adopt the following rule. For all $\widetilde{h}(t) \in\left[-S(t), S^{*}(t)\right]$, we define $\widetilde{h}(t)=$ $\widetilde{h}(t-\Delta t)+x(t-\Delta t)$, where

$$
\widetilde{h}(t)=\left\{\begin{array}{l}
h(t) \in[-S(t), O(t)] \\
h^{*}(t) \in\left[O(t), S^{*}(t)\right]
\end{array}\right.
$$

and $O(t)$ stands for the origin at period $t$, i.e., $O(t)=0$, for all $t \geq 0$.

Thus, we assume that the preferences for the home location will change in the following way. For the firms that do not move, attachment to home will increase by $x$ if the small economy is attractive to foreign investors $(-x<0)$ and it will decrease if the foreign location attracts capital from the small jurisdiction $(x>0)$. For the capital owners who move abroad, the higher their attachment to the jurisdiction they leave, the lower the attachment to the new location will be. Notice that, interestingly, in our setting home attachment is endogenous and modifiable by the economic policy, similarly to Han et al (2014).

In the rest of the paper we focus on the small jurisdiction. We analyze, in an infinite time horizon, the dynamics of its economic size $S(t)$ and its policy instruments $T(t)$ and $a(t)$ for exogenous foreign levels of taxes $T^{*}$ and public goods $a^{*}$. So, we consider that the rest of the world does not react to the small jurisdiction's decisions. We also analyze the convergence of the variables $S(t), T(t)$ and $a(t)$ toward possible steady states.

Suppose that policy makers maximize the discounted linear-quadratic utility that depends on tax revenues, $S(t) \cdot T(t)$, net of the cost of producing public inputs $\beta / 2 a^{2}(t)$, where $\beta>0$ represents an efficiency parameter. The objective-function of the small economy is given by

$$
\begin{aligned}
\max _{T(t)} W= & \int_{0}^{\infty} e^{-r t}\left[S(t) T(t)-\frac{\alpha}{2}(S(t) T(t))^{2}-\frac{\beta}{2} a^{2}(t)\right] d t, \\
& \text { subject to } \\
\dot{a}(t)= & -\delta a(t)+\zeta S(t) T(t), \\
\dot{S}(t)= & -x=\frac{a(t)-T(t)}{k}-b^{*}(t), \\
0 \leq & S(t)<\bar{S}<\frac{1}{2}
\end{aligned}
$$

where $\alpha$ represents a cost parameter of collecting taxes ${ }^{13}$ and the rate of time preference $r$ which represents the degree of "impatience" of the home population. We assume furthermore that the linear-quadratic utility is increasing and concave with respect to the total tax, that is, $\alpha$ is such that $T<1 / \alpha$.

\footnotetext{
${ }^{13}$ Collecting tax is costly, see for example, Vaillancourt, 1989.
} 
We now characterize the inter-temporal optimal tax strategy chosen by the policy makers in the small jurisdiction. Applying Pontryagin's maximum principle, we derive a canonical system of ordinary differential equations that has to be satisfied by the optimal trajectories. Since the maximized Hamiltonian of the dynamic optimization problem is concave with respect to the state variables, the Maximum principle provides not only necessary but also sufficient optimality conditions for interior solutions (see e.g. Theorem 4.2, Dockner et al. ,2000, Chiang, 2000, or Hartl et al.,1995). Denote by $\mu$ and $v$ the costate variables corresponding respectively to the state variables $S(t)$ and $a(t)$, we conclude the following findings.

Proposition 1 For any state trajectory $(S(t), a(t))$, there exist piecewise absolutely continuous co-states $\mu(t)$ and $\nu(t)$, and two multipliers $\theta_{1}(t) \geq 0, \theta_{2}(t) \geq 0$, such that the optimal choice variable $T(t)$ satisfies

$$
T(t) S^{2}(t)=\frac{(1+\zeta \nu) S-\frac{1}{k}\left(\mu-\theta_{1}+\theta_{2}\right)}{\alpha} .
$$

The costate equations become

$$
\begin{aligned}
\dot{\mu} & =r \mu-\zeta T \nu-(1-\alpha T S) T \\
\dot{\nu} & =(r+\delta) \nu+\beta a-\frac{1}{k}\left(\mu-\theta_{1}+\theta_{2}\right) .
\end{aligned}
$$

Furthermore,

$$
\begin{aligned}
& \dot{S}=-\left(b^{*}-\frac{a-T}{k}\right) \leq 0, \text { if } S(t)=\frac{1}{2} ; \theta_{1}(t)\left(\frac{1}{2}-S(t)\right)=0, \theta_{1} \geq 0, \\
& \dot{S}=-\left(b^{*}-\frac{a-T}{k}\right) \geq 0, \text { if } S(t)=0 ; \theta_{2}(t) S=0, \theta_{2} \geq 0 .
\end{aligned}
$$

Finally, the transversality conditions $\lim _{t \rightarrow \infty} e^{-r t} \mu S=0$ and $\lim _{t \rightarrow \infty} e^{-r t} \nu a=0$ are satisfied.

In the following section, we characterize the potential steady states of the system and we analyze how the steady states can be attained.

\section{Steady states and convergence}

Steady states are defined as rest points of the dynamic equations (2), (6), (11) and (12) remembering that the decision variables $\left(a^{*}\right.$ and $\left.T^{*}\right)$ of the rest of the world are given. Due to the state space constraints, there are two types of possible long term solutions: efficient steady state(s) and inefficient (constrained) steady states. In the first case, the small jurisdiction survives in the long run without 
attaining its maximum economic size $(0<\widehat{S}<\bar{S})$ or it reaches its highest possible size $\left(\widehat{S}=\bar{S}<\frac{1}{2}\right)$. In the inefficient case, the small economy can eventually collapse $(\underline{S}=0)$ or it expands to the limit level $\bar{S}<\frac{1}{2}$. Note that in the first scenario, the upper-limit solution is efficient while it is not in the second scenario. As it will be clear in the following sections, in the upper-limit solution of the constrained steady state, the solution is inefficient because the constraint on the state variable becomes binding Hence, if the small economy reaches its upper bound $\bar{S}$ in the constrained steady state, additional FDIs can no more be absorbed.

\subsection{The small economy converges to an efficient size}

The existence of an efficient steady state is crucial. Indeed, it means that there exists a policy mix consisting of taxes and public infrastructures able to guarantee the long term survival of a small economy. If we consider interior steady states $\widehat{S}(0<\widehat{S}<\bar{S})$, the boundary constraints are both not binding. Hence, $\hat{\theta}_{1}=0$ and $\hat{\theta}_{2}=0$ and the interior rest points of the dynamic system (2), (6), (11) and (12) are specified in the following proposition, the proof of which is given in the appendix. The following proposition states that there exists a unique efficient steady state for any parameter constellation.

Proposition 2 Assume foreign policy choices are $a^{*}$ and $T^{*}$. There is always one ${ }^{14}$ steady state given by

$$
\hat{a}=\frac{\zeta(r+\delta)}{\alpha(r+\delta) \delta+\beta \zeta^{2}}(>0), \hat{S}=\frac{\delta}{\zeta} \frac{\hat{a}}{\widehat{T}}, \text { and } \hat{T}=\hat{a}-\left(a^{*}-T^{*}\right)
$$

and the two costate variables are

$$
\widehat{\mu}=0 \text { and } \widehat{\nu}=-\frac{\beta}{r+\delta} \hat{a}(<0)
$$

This steady state is a saddle point of the canonical system (2), (6), (11) and (12). Moreover, if $r>\frac{\widehat{T}}{k \widehat{S}^{\prime}}$ the convergent path is a one dimensional manifold. Otherwise, if $r<\frac{\widehat{T}}{k \widehat{S}}$, the convergent path is a two dimensional manifold.

In our model, we have a four dimensional steady state attainable either by a one dimensional path or by a two-dimensional path. ${ }^{15}$ The first case occurs when in the four-dimensional eigenvalue

\footnotetext{
${ }^{14}$ Notice that in addition to the above interior steady state, other interior solutions may appear for special parameter and coefficient combinations. Because these cases correspond to restricted conditions, we present them in the Appendix.

${ }^{15}$ The existence of a variety of dimensional convergence paths is not new in the literature relative to saddle point stability. For example, in the Lorenz system (see Guckenheimer, 2002), there exists a three dimensional steady state
} 
space, there exists only one negative eigenvalue. The second case occurs when there are two negative eigenvalues. When there is an one-dimensional convergence path, long run efficiency is only attainable by chance because we have two "non-jump" variables $S(t)$ and $a(t)$ with given initial size $S(0)$ of the small economy and its initial level of infrastructure $a(0) .{ }^{16}$ This unfavorable case occurs when $r>\frac{\widehat{T}}{k \widehat{S}}$. However, when $r<\frac{\widehat{T}}{k \widehat{S}}$, the small economy will be on the convergence path leading to the efficient steady state for any initial conditions given by the predetermined pair $(S(0), a(0)){ }^{17}$

In the following we show that the convergence to the long run values $\hat{S}$ and $\widehat{T}$ depends on the share $\zeta$ of tax revenue allocated to attractive infrastructure. More technically, in Appendix A.3, we demonstrate that the small economy converges to the efficient steady state if $r<\frac{\widehat{T}}{k \widehat{S}^{\prime}}$, which is equivalent to $k<\frac{g^{2} \alpha}{r}\left(g=a^{*}-T^{*} \neq 0\right)$ and $\zeta \in(0, \min (\underline{\zeta}, 1)), \underline{\zeta}>0$. However, if capital mobility is not high enough $\left(k>\frac{g^{2} \alpha}{r}\right)$, convergence is not necessarily guaranteed. The following proposition states this result.

Proposition 3 When capital mobility is high enough there exists a non-empty range of tax shares $\zeta$ allocated to local infrastructure that allow the small jurisdiction to converge to its long run efficient state. When capital mobility is too low, the attainment of an efficient steady state is not guaranteed.

The policy implication of this proposition is straightforward. If there is sufficient capital mobility, the survival of the small jurisdiction is in the hands of its decision makers. The share of taxes allocated to infrastructure expenditures is a crucial one-shot policy instrument to guarantee the convergence to an efficient steady state.

The following corollary can be stated.

Corollary 4 In case of perfect capital mobility $(k=0)$, the efficient steady state is always attainable for any initial conditions.

Let us now analyze the nature of the steady state solutions. First note that according to (14) it is optimal for the small state to equate the net (of taxes) amount of provided public goods $\widehat{a}-\widehat{T}$ to that of the foreign economy $a^{*}-T^{*}$. This equality is necessary for migration to cease. Furthermore, the

but according to different parameter constellations this equilibrium can be attained by one or two-dimensional paths. Similarly, Krauskopf et. al.(2008) provide a numerical simulation of the same concepts and other authors like Kiyotaki and Moore (1997) and Eicher and Turnovsky (2001) provide economic applications.

${ }^{16}$ This particular result is consistent with Blanchard and Kahn (1980). Then convergence to the steady state only can occur if the two state variables $S(t)$ and $a(t)$ are generated by the eigenvector which corresponds to the unique negative eigenvalue. Otherwise, there is explosion in the sense of Blanchard and Kahn (1980). Mathematically, the convergent path is a zero measure subset (here it is a line) in a two dimensional state-space.

${ }^{17}$ The tax rate of the small country $T$ is the (jump) adjustable variable which ensures that the economy will be initially on the saddle-point path. 
amount of public infrastructure offered in the steady state does not directly depend on the decision variables of the rest of the world. This is not the case for the equilibrium tax rate $\widehat{T}$ and consequently for the equilibrium size $\widehat{S}$. In other words, the equilibrium level of infrastructures provided by the small state depends only on parameters specific to its economy, while the equilibrium tax level is both determined by domestic and foreign characteristics. ${ }^{18}$ At first sight it is remarkable that the small economy is independent from international conditions in determining its long term public infrastructures. The underlying intuition is however straightforward. Assume the large economy becomes more attractive which means that $\left(a^{*}-T^{*}\right)$ increases. In order to preserve its attractiveness, the small economy reacts by decreasing its tax rate $\widehat{T}$ in a way to keep unchanged the provision of its infrastructure.

Comparative statics We can now turn to some comparative statics. We see that the steady state provision of public goods increases with the time preference $r\left(\frac{\partial \widehat{a}}{\partial r}>0\right)$. The reason is that the more the home jurisdiction is impatient the more it will be reluctant to postpone expenditures in public infrastructures. However, the impact of a higher share $\zeta$ of tax revenue allocated to infrastructures by the small economy is ambiguous. Indeed, according to (14), we have $\frac{\partial \widehat{a}}{\partial \zeta}>0$ if $\zeta<\bar{\zeta}\left(\bar{\zeta}=\sqrt{\frac{\alpha \delta(r+\delta)}{\beta}}\right.$ ) and $\frac{\partial \widehat{a}}{\partial \zeta}<0$ if $\zeta>\bar{\zeta}$. Therefore, as long as $\zeta<\bar{\zeta}$, allocating more (increasing $\zeta$ ) tax revenue to the provision of public inputs increases the equilibrium level of infrastructures and the equilibrium tax level. ${ }^{19}$ In other words, the small jurisdiction reacts by increasing its infrastructure attractiveness and by decreasing its tax competitiveness. However, if the threshold $\bar{\zeta}$ is exceeded, devoting a larger share of taxes to public inputs paradoxically decreases the equilibrium level of infrastructures. The reason is that increasing $\zeta$ above $\bar{\zeta}$ induces too high infrastructure expenditures for a given level of taxes. The small state then prefers to switch to the opposite regime of lowering its infrastructure attractiveness and augmenting its tax competitiveness (decreasing $\widehat{T}$ ). Also note that the likeliness of this last regime choice increases with the inefficiency in providing infrastructures, because $\bar{\zeta}$ decreases with $\beta$.

According to (14) we have $\widehat{S} \widehat{T}=\frac{\delta \widehat{a}}{\zeta}$, which means that the tax income of the small jurisdiction is not affected by changes in the net attractiveness $\left(a^{*}-T^{*}\right)$ of the rest of the world. For example, if $a^{*}-T^{*}$ rises, the home jurisdiction will react by decreasing its equilibrium tax rate $\widehat{T}$. This will attract new foreign firms and will increase $\widehat{S}$ in such a way that the tax income $\widehat{S} \widehat{T}$ remains unchanged.

Next we analyze how a change ${ }^{20}$ in $\widehat{a}$ impacts the equilibrium size of the home economy. It is

\footnotetext{
${ }^{18}$ An exception could be the parameter $r$ if it is interpreted as an international rate of interest, but this is clearly not the case in our model.

${ }^{19}$ Since $\widehat{T}=\widehat{a}-\left(a^{*}-T^{*}\right)$, and thus $\frac{\widehat{\partial a}}{\partial \zeta}$ and $\frac{\partial \widehat{T}}{\partial \zeta}$ are equally signed.

${ }^{20}$ This change can result from a shock on $\zeta$ or $r$.
} 
straightforward to show that $\frac{\partial \widehat{S}}{\partial \widehat{a}}$ has the opposite $\operatorname{sign}^{21}$ of $a^{*}-T^{*}$. Because the condition $\widehat{T}=$ $\widehat{a}-\left(a^{*}-T^{*}\right)$ must hold in the steady state, both world-regions are equally attractive if the net amount of public goods offered by the small and large economies are either positive $\left(a^{*}>T^{*}\right.$ and $\left.\widehat{a}>\widehat{T}\right)$. Conversely, both regions are equally unattractive if $T^{*}>a^{*}$ and $\widehat{T}>\widehat{a}$. The derivative $\frac{\partial \widehat{S}}{\partial \widehat{a}}$ is negative in the first case and positive in the second case. The impact of $\widehat{a}$ on $\widehat{S}$ can be interpreted in the following way. If both regions are equally attractive, entrepreneurs have (for given moving costs) a preference for the jurisdiction which lowers it tax rate. However, if the small jurisdiction increases the provision of public inputs it increases its tax rate (see equation (15)). It follows that capital flows out of the small jurisdiction and the size $\widehat{S}$ shrinks. If both regions are equally unattractive, entrepreneurs have (for given moving costs) a preference for the region which increases the provision of infrastructure. Hence, an increase in $\widehat{a}$ results in a capital inflow and the size $\widehat{S}$ expands consequently.

Until now we answered the question about the capacity of the small economy to survive in an efficient way. However, economic viability can occur in two different ways. Relative to its initial economic size, it can shrink $(\widehat{S}<S(0))$ or it can extend $(\widehat{S}>S(0))$. In the latter case, it can reach its maximal attainable size if the parameter combination $\left(\delta, \zeta, k, r\right.$ and $\left.a^{*}, T^{*}\right)$ is such that $\widehat{S}=\bar{S}$

If the policy makers of the small jurisdiction are able to set the economy on the right path, their economy will survive in the long run, otherwise the economy will diverge. Divergence will end up in one of two inefficient steady states we analyze in the following section. The inefficient long run outcome can be either the upper-bound solution $\bar{S}$ or economic collapse $(\underline{S}=0)$.

\subsection{The small economy diverges to inefficient steady states}

\subsubsection{Divergence to the upper-bound}

We saw above that there is either a one dimensional or a two-dimensional trajectory that converges to the optimal steady state. All other trajectories lead to one of the two inefficient solutions; $\underline{S}=0$ and $\bar{S}<\frac{1}{2} \cdot{ }^{22}$ Let us now consider the case in which the small jurisdiction diverges to its upper bound $S(t)=\bar{S}$. Remember that we realistically assume that the small economy is limited in its capacity to grow bigger than the rest of the world. Even if it reaches its upper bound size it will remain small enough to consider the rest of the world's choices as exogenously given.

If $S(t)=\bar{S}<\frac{1}{2}$, the constraint on the state variable becomes binding and the steady state value of $\theta_{1}$ has to be positive and $\theta_{2}=0$. Hence, condition (13) in Proposition 1 should hold. If the small

$21 \frac{\partial \widehat{S}}{\partial \widehat{a}}=-\frac{1}{\zeta} \frac{\delta}{\left(\widehat{a}+T^{*}-a^{*}\right)^{2}}\left(a^{*}-T^{*}\right)$

${ }^{22}$ For more discussion on the saddle point stability see, for example, Eicher and Turnovsky (2001) or Galor (2007), and the references therein. 
economy reaches its upper bound $\bar{S}$, additional FDIs can no more be absorbed and formally, $S(t)$ is constant or decreasing: $(\dot{S}(t) \leq 0)$. In the appendix, we show how the following steady state values are obtained

$$
\bar{S}=\bar{S}<\frac{1}{2}, \bar{a}=\frac{\zeta}{\zeta-2 \delta}\left(a^{*}-T^{*}\right), \bar{T}=\bar{a}-\left(a^{*}-T^{*}\right) .
$$

The corresponding costate variables are

$$
\begin{gathered}
\bar{\nu}=\frac{1}{r+\delta-\frac{\zeta}{2}}\left(\frac{1}{2}-\frac{\alpha \bar{T}}{4}-\beta \bar{a}\right) \text { and } \bar{\mu}=\frac{\bar{T}}{r}\left(1-\frac{\alpha \bar{T}}{2}+\zeta \bar{\nu}\right), \\
\bar{\theta}_{1}=\left(1-\frac{2 k r}{\rho \bar{T}}\right) \bar{\mu}>0, \text { and } \bar{\theta}_{2}=0 .
\end{gathered}
$$

The following proposition can be stated

Proposition 5 The economy converges to its upper-limit size, given by (17), if (i) $\zeta>2 \delta>0$ and $a^{*}>T^{*}$, or (ii) $0<\zeta<2 \delta$ and $a^{*}<T^{*}$. The multiplier $\theta_{1}$ is given by (19) and is strictly positive with $\mu$ and $\nu$ given respectively by (18).

According to equation (17), we see that if the small jurisdiction's economy converges to its limitsize $\bar{S}<\frac{1}{2}$, it has to tie its long term policy decisions to $\left(a^{*}-T^{*}\right)$ according to a linear rule ${ }^{23}$. When the net benefit offered by the rest of the world is positive, $a^{*}-T^{*}>0$ (case a), it appears that the home jurisdiction can converge to the upper-bound value $\bar{S}<\frac{1}{2}$ if the share of tax revenue devoted to infrastructure expenditures, $\zeta$, is high enough $(\zeta>2 \delta>0)$.

\subsubsection{Possible collapse}

Now we consider the case where the small economy could suffer from a possible economic collapse, i.e., $S(t)=0$. In this case, the constraint on the state variable must be binding in order to exclude a negative population value and the steady state values of $\theta_{1}=0$ and $\theta_{2}$ have to be positive. Hence, condition (14) in Proposition 1 should hold. Furthermore, once $S(t)$ has attained the lower bound, it can no more decrease and formally, it should be either constant or increasing, that is, $\dot{S}(t) \geq 0$, as it is shown in Proposition 1. In this case, we get

$$
\begin{aligned}
& \underline{S}=0, \underline{\theta_{1}}=0, \underline{\theta_{2}}=-\mu, \\
& \underline{a}=0, \underline{T}=T^{*}-a^{*}, \\
& \underline{\nu}=0, \underline{\mu}=\frac{1}{r}\left(T^{*}-a^{*}\right)(<0) .
\end{aligned}
$$

Hence, the following result obtains.

\footnotetext{
${ }^{23}$ Indeed, from (21) we deduce $\bar{T}=\frac{2 \zeta}{\zeta-2 \delta}\left(a^{*}-T^{*}\right)$.
} 
Proposition 6 Assume that the policy mix of the rest of the world is such that, $T^{*}<a^{*}{ }^{24}$ Then, the small state is heading for collapse $(\underline{S}=0)$ specified in (20). The multiplier $\theta_{2}$ is strictly positive.

It follows that the economy of the small jurisdiction may only collapse if the rest of the world is able to provide a positive net public benefit $\left(a^{*}-T^{*}>0\right)$. In other words,

Corollary 7 The small economy never collapses if the rest of the world is not attractive $\left(a^{*}-T^{*}<0\right)$.

Moreover, if the small jurisdiction is caught on a path leading to a long run collapse, even aggressive tax dumping will not be able to curb steady capital flight. Eventually, the small jurisdiction's tax and infrastructure expenditures will vanish.

\section{Conclusion}

Many authors recognize that small jurisdictions lack (quantitatively and qualitatively) fundamental productive resources. These deficiencies appear especially in the form of limited productive capital, entrepreneurs and human capital. In this paper, for tractability, we merged these three types of production factors in one entity by assuming that a capital owner, a firm owner and a worker are the same person. Then, we analyzed how an appropriate policy-mix, implemented by the small economy, was able to attract these lacking resources. Applying dynamic optimization techniques, we derived efficient and inefficient steady states and their stability conditions. We show that the share of tax income attributed by the small economy to infrastructure expenditures plays a key-role in ensuring an efficient convergence. A neglect of this aspect can be deleterious for the survival of a small economy.

In this paper, the focus was on a small economy which takes the policy decisions of the foreign world as given. This assumption is generally acceptable if the jurisdiction is small. In a future work the analytical framework we developed in this paper should be extended to a non-cooperative game between the small and the big jurisdictions. Accordingly, the question of a likely economic collapse affecting the small economy could be reassessed.

\footnotetext{
${ }^{24}$ This is only necessary condition but not sufficient.
} 


\section{A Appendix}

\section{A.1 Proof of Proposition 1}

The current value of the Hamiltonian corresponding to the underlying economy is

$$
\mathcal{H}(T, S, a, \mu, \nu)=\left[S T-\frac{\alpha}{2}(S T)^{2}-\frac{\beta a^{2}}{2}\right]-\mu\left(b^{*}(t)-\frac{a(t)-T(t)}{k}\right)+\nu[-\delta a(t)+\zeta S T] .
$$

The following Lagrangian accounts for the state constraint ${ }^{25}$ of the model

$$
\mathcal{L}\left(T, S, a, \mu, \nu, \theta_{1}, \theta_{2}\right)=\mathcal{H}(T, S, a, \mu, \nu)+\theta_{1}\left(b^{*}(t)-\frac{a(t)-T(t)}{k}\right)-\theta_{2}\left(b^{*}(t)-\frac{a(t)-T(t)}{k}\right) .
$$

It is easy to see that $\mathcal{H}(T, S, a, \mu, \nu)$ is concave with respect to the state variables $S$ and $a$. Hence, the first order conditions are necessary and sufficient for the existence of an optimum. Deriving the first order conditions from the Hamiltonian we obtain (10) with respect to $T$, while we get (12) and (11) with respect to both state variables. The multipliers of the state boundary constraints check (13) and (14). QED.

\section{A.2 Proof of Proposition 2- Existence of Equilibria}

We first state the existence of possible interior steady state(s) in addition to that given in Proposition 2. Then we provide the proof.

Proposition 8 The following additional steady state(s) may appear:

(II.1) If $a^{*}-T^{*}=-\frac{k r \delta}{4 \zeta}(<0)$, there is a further interior steady state specified by

$$
\widehat{T}_{1}=\frac{k r \delta}{2 \zeta}, \widehat{a_{1}}=\left(a^{*}-T^{*}\right)+\widehat{T}_{1}, \widehat{S}_{1}=\frac{\widehat{T}_{1}}{k r}
$$

and two costate variables ${ }^{26}$

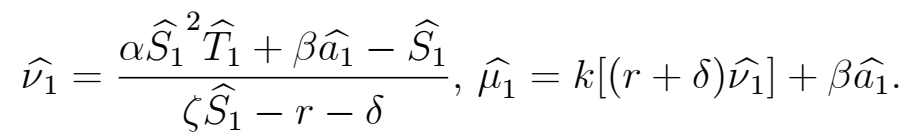

(II.2) If $a^{*}-T^{*}=0$, the additional steady state is

$$
\widehat{T}_{2}=\frac{k r \delta}{\zeta}
$$

\footnotetext{
${ }^{25}$ See, for example, Chiang, page 301-302.

${ }^{26}$ The condition $\zeta \widehat{S_{1}}-r-\delta+\xi \neq 0$ must hold.
} 
and we obtain the remaining steady state variables by replacing the subscript 1 by 2 in (23) and (24).

(II.3) If $a^{*}-T^{*}>0$, the second steady state is specified by

$$
\widehat{T}_{3}=\frac{k r}{2 \zeta}\left[\delta+\sqrt{\delta^{2}+\frac{4 \zeta}{k r} \delta\left(a^{*}-T^{*}\right)}\right]
$$

and the others are the same as in (23) and (24) by replacing the subscript 1 to 3.

(II.4) If $-\frac{k r \delta}{4 \zeta}<a^{*}-T^{*}<0$, there are another two interior steady states where

$$
\widehat{T_{4,5}}=\frac{k r}{2 \zeta}\left[\delta \pm \sqrt{\delta^{2}+\frac{4 \zeta}{k r} \delta\left(a^{*}-T^{*}\right)}\right]
$$

and the remaining the others are the same as in (23) and (24) by replacing the subscript 1 by 4 and 5.

\section{Proof.}

At the interior steady state, $\theta_{1}=0, \theta_{2}=0$ and we can rewrite the first order condition as follows

$$
\left\{\begin{array}{l}
T=\frac{S-\frac{\mu}{k}+\zeta S \nu}{\alpha S^{2}} \\
\dot{S}=-\left(b^{*}-\frac{a-T}{k}\right), \\
\dot{a}=\delta a+\zeta S T, \\
\dot{\mu}=r \mu-\zeta T \nu-(1-\alpha S T) T, \\
\dot{\nu}=(r+\delta) \nu+\beta a-\frac{1}{k} \mu .
\end{array}\right.
$$

We rewrite the first equation as follows

$$
\frac{\mu}{k S}=1+\zeta \nu-\alpha S T \text {. }
$$

Substituting (29) into the 3rd equation and arranging leads to the

$$
\dot{\mu}=r \mu-\frac{T \mu}{k S} \text {. }
$$

Hence, $\dot{\mu}=0$ leads to two cases: $\widehat{\mu}=0$ or $r=\frac{\widehat{T}}{k \widehat{S}}$.

We consequently have two groups of steady states: general one $\widehat{\mu}=0$ or/and special one where $r=\frac{\widehat{T}}{k \widehat{S}}$.

(I) $\widehat{\mu}=0$.

It is easy to check that the interior steady states are given by (15) and (16). To determine their stability, we consider the corresponding Jacobian 


$$
\begin{aligned}
& J_{I}=\left(\begin{array}{cccc}
\frac{1}{k \alpha} \frac{1+\zeta \widehat{\nu}}{\widehat{S}^{2}} & \frac{1}{k} & \frac{1}{\alpha k^{2} \widehat{S}^{2}} & -\frac{\zeta}{\alpha k \widehat{S}} \\
0 & -\delta & -\frac{\zeta}{\alpha k \widehat{S}} & \frac{\zeta^{2}}{\alpha} \\
0 & 0 & -\frac{1}{k \alpha} \frac{1+\zeta \widehat{\nu}}{\widehat{S}^{2}}+r & 0 \\
0 & \beta & -\frac{1}{k} & r+\delta
\end{array}\right) \\
& =\left(\begin{array}{cccc}
\frac{\widehat{T}}{k \widehat{S}} & \frac{1}{k} & \frac{1}{\alpha k^{2} \widehat{S}^{2}} & -\frac{\zeta}{\alpha k \widehat{S}} \\
0 & -\delta & -\frac{\zeta}{\alpha k \widehat{S}} & \frac{\zeta^{2}}{\alpha} \\
0 & 0 & -\frac{\widehat{T}}{k \widehat{S}}+r & 0 \\
0 & \beta & -\frac{1}{k} & r+\delta
\end{array}\right) .
\end{aligned}
$$

It is easy to show that the eigenvalues of the Jacobian are given by

$$
\begin{aligned}
& e_{1}=\frac{\widehat{T}}{k \widehat{S}}>0, \quad e_{2}=r-\frac{\widehat{T}}{k \widehat{S}}>0(\text { or }<0), \\
& e_{3,4}=\frac{r}{2} \pm \frac{1}{2} \sqrt{r^{2}+4\left[\frac{\beta \zeta^{2}}{\alpha}+(r+\delta) \delta\right]} .
\end{aligned}
$$

Hence, $e_{3}>0$ and $e_{4}<0$, which guarantees one dimensional convergence to the steady state. The other part of the convergence depends on $e_{2}$ is negative or not, that is, the relation of $r$ with respect to the other parameters and exogenous variables.

(II) $r=\frac{\widehat{T}}{k \widehat{S}}$.

In this case, we have $S=\frac{T}{k r}$. $\dot{S}=0$ leads to $a=\left(a^{*}-T^{*}\right)+T$ and $\dot{a}=0$ gives $\zeta S T=\delta a$. Combining these conditions, we obtain

$$
\frac{\zeta}{k r} T^{2}-\delta T-\delta\left(a^{*}-T^{*}\right)=0
$$

which yields to two roots

$$
T=\frac{k r}{2 \zeta}\left[\delta \pm \sqrt{\delta^{2}+\frac{4 \zeta}{k r} \delta\left(a^{*}-T^{*}\right)}\right]
$$

and if $\Lambda=\delta^{2}+\frac{4 \zeta}{k r} \delta\left(a^{*}-T^{*}\right)>0$, both roots are real. Furthermore, depending on $\Lambda$ is larger or smaller than $\delta^{2}$, we have different conditions which leads to positive $T$ s and which serve as the other steady states. 


\section{A.3 Proof of Proposition 2- convergence and Corollary 1}

After taking account of

$$
\hat{a}=\frac{\zeta(r+\delta)}{\alpha(r+\delta) \delta+\beta \zeta^{2}}(>0), \hat{S}=\frac{\delta}{\zeta} \frac{\hat{a}}{\widehat{T}}, \hat{T}=\hat{a}-\left(a^{*}-T^{*}\right)
$$

and noting that $g=a^{*}-T^{*} \neq 0$, the condition $r<\frac{\widehat{T}}{k \widehat{S}}$ is equivalent to $\Omega(\zeta)<0$, where

$$
\begin{aligned}
\Omega(\zeta) & =\left(-g \beta^{2}\right) \zeta^{4}+2 g \beta(r+\delta) \zeta^{3}-(r+\delta)(r+\delta-k r \beta \delta+2 g \alpha \beta \delta) \zeta^{2} \\
& +\left(2 g \alpha \delta(r+\delta)^{2}\right) \zeta-\alpha \delta^{2}(r+\delta)^{2}\left(g^{2} \alpha-k r\right)
\end{aligned}
$$

is a 4th order polynomial in $\zeta$, with $\Omega(\zeta=0)=-\alpha \delta^{2}(r+\delta)^{2}\left(g^{2} \alpha-k r\right)<0$ when $k<\frac{g^{2} \alpha}{r}$.

If we only consider the case of real roots of $\Omega(\zeta)=0$, Desecrates's rule of signs guarantees that the polynomial $\Omega(\zeta)$ has at least two positive real roots. Let us denote by $\underline{\zeta}$ the smallest positive root of $\Omega(\zeta)=0$. If $\underline{\zeta}<1$, the polynomial $\Omega(\zeta)$ is negatively signed for $\zeta \in(0, \underline{\zeta})$ when $k<\frac{g^{2} \alpha}{r}$. If $\underline{\zeta} \geq 1$, we have $\Omega(\zeta)<0$ for all $\zeta \in(0,1)$ when $k<\frac{g^{2} \alpha}{r}$. Nonetheless, $k>\frac{g^{2} \alpha}{r}$ yields $\Omega(\zeta=0)>0$ and the polynomial $\Omega(\zeta)$ is negatively signed for all $\zeta \in(\widetilde{\zeta}, 1)$ iff $\widetilde{\zeta}<1$, where $\widetilde{\zeta}$ is the first positive root of $\Omega(\zeta)=0$. In other words if $\widetilde{\zeta} \geq 1, \Omega(\zeta)>0$ for all $\zeta \in(0,1)$, the efficient long term state is only attainable by chance.

That completes the proof of Proposition 2.

The proof of Corollary 1 derives from the fact that, $\Omega(\zeta=0)=-\alpha \delta^{2}(r+\delta)^{2}\left(g^{2} \alpha-k r\right)<0$ when $k<\frac{g^{2} \alpha}{r}$. Thus, it is straightforward that, $k<\frac{g^{2} \alpha}{r}$ and

$\zeta \in(0, \min (\underline{\zeta}, 1))$ imply $r<\frac{\widehat{T}}{k \widehat{S}}$.

\section{A.4 Proof of Proposition 5}

In the upper-corner solution case, $S=\frac{1}{2}$ and, hence, $\theta_{2}=0$ and $\theta_{1}>0$. According to the complementary slackness conditions, we must have $\bar{a}-\bar{T}=a^{*}-T^{*}$, that is, $\bar{T}=\bar{a}-\left(a^{*}-T^{*}\right)$.

From $\dot{a}=0$, we obtain

$$
\bar{a}=\frac{\frac{\zeta}{2}\left(a^{*}-T^{*}\right)}{\frac{\zeta}{2}-\delta} .
$$

The solution $\bar{a}$ is be positive if and only if $\delta>\frac{\zeta}{2}$ and $a^{*}<T^{*}$, or $0<\delta<\frac{\zeta}{2}$ and $a^{*}>T^{*}$. 
The condition $\dot{\mu}=0$ leads to

$$
r \bar{\mu}=\frac{\bar{T}}{2} \frac{1}{k}\left(\bar{\mu}-\overline{\theta_{1}}\right)
$$

or

$$
\overline{\theta_{1}}=\left(\frac{2 k r}{\bar{T}}-1\right) \bar{\mu} .
$$

Similarly, $\dot{\nu}=0$ leads to

$$
(r+\delta) \bar{\nu}=\frac{1}{k}\left(\bar{\mu}-\overline{\theta_{1}}\right)-\beta \bar{a} .
$$

On the other hand, the first order condition with respect to $T$ can be rewritten as

$$
\alpha \bar{S} \bar{T}=1-\frac{1}{k} \frac{\bar{\mu}-\theta_{1}}{\bar{S}}+\zeta \bar{\nu}
$$

which gives

$$
-\frac{1}{k}\left(\bar{\mu}-\overline{\theta_{1}}\right)=\frac{\alpha \bar{T}}{4}-\frac{1}{2}-\frac{\zeta \bar{\nu}}{2} .
$$

Combining with (33), it follows

$$
\bar{\nu}=\frac{1}{r+\delta-\frac{\zeta}{2}}\left[\frac{1}{2}-\beta \bar{a}-\frac{\alpha \bar{T}}{4}\right]
$$

and

$$
\bar{\mu}=\frac{\bar{T}}{r}\left(1+\zeta \bar{\nu}-\frac{\alpha \bar{T}}{2}\right) .
$$

Hence, we obtain a complete solution for the steady state $\bar{S}<\frac{1}{2}$. The above solutions are meaningful if and only if $\overline{\theta_{1}}>0$.

\section{References}

[1] Alesina, A., E. Spolaore, and R. Wacziarg (2005). Trade, growth and the size of countries. In Handbook of Economic Growth, P. Aghion and Durlauf, S. 1499-1542: North Holland, Amsterdam.

[2] Armstrong, H., R. J. De Kervenoael, X. Li, and R. Read (1998). A comparison of the economic performance of different micro-states, and between micro-states and larger countries. World Development, 26(4): 639-656.

[3] Armstrong, H. and R. Read (1995). Western European micro-states and EU autonomous regions: The advantages of size and sovereignty. World Development, Vol. 23 No. 7, pp. 1229-1245. 
[4] Armstrong, H. W. and R. Read (2002). The phantom of liberty?: economic growth and the vulnerability of small states. Journal of International Development (14), 435-458

[5] Basu A., M. Casson, N. Wadeson, and B. Yeung (2008). The Oxford Handbook of Entrepreneurship. Oxford University Press.

[6] Behrens K. (2005). Market size and industry location: Traded vs. non-traded goods. Journal of Urban Economics, 58, 24-44

[7] Blanchard, O.J., and C.M. Kahn (1980). The solution of linear difference models under rational expectations. Econometrica 48, 1305-1311.

[8] Blumenthal, M. and J. Slemrod (1992). The compliance cost of the U.S. individual tax system: a second look after tax reform. National Tax Journal, 45(2), 186-202.

[9] Boucekkine R., C. Le Van and C. Saglam (2007). Optimal control in infinite horizon problems: A Sobolev Space approach. Economic Theory, 32, 497-509.

[10] Briguglio L. (1995). Small island developing states and their economic vulnerabilities. World Development 23(9), 1615-1632.

[11] Briguglio , L., G. Cordina, N. Farrugia and S. Vella (2009). Vulnerability in development: advances in concept and measurement, Oxford Development Studies, Vol. 37, No. 3, 229 - 247

[12] Cardarelli, R., Taugourdeau, E. and J. P. Vidal(2002). A repeated interactions model of tax competition. Journal of Public Economic Theory 4, 19-38.

[13] Castellani D. and A. Zanfei (2006). Multinational firms, innovation and productivity. Edward Edgar Publishing Limited.

[14] Catenaro, M. and J. P. Vidal, (2006). Implicit tax coordination under repeated policy interactions. Recherches Economiques de Louvain 72, 1-17.

[15] Chiang, A. (2000). Elements of dynamic optimization. Waveland Press, Illinois.

[16] Dockner, E. J., S. Jorgensen , N.V. Long and G. Sorger (2000). Differential games in economics and management science, Cambridge. Cambridge University Press.

[17] Eicher T. and S. Turnovsky (2001). Transitional dynamics in two-sector non-scale growth model. Journal of Economics and Control, 25, 85-112. 
[18] Galor O. (2007), Discrete dynamical system. Springer.

[19] Guckenheimer J. (2002). Numerical analysis of dynamic system. Chapter 8 of Handbook of Dynamical System, Edited by B. Fiedler, vol. 2, Elsevier, 345-390.

[20] Haufler A. (1997). Factor taxation, income distribution and capital market integration. Scandinavian Journal of Economics, Wiley Blackwell, 99(3), 425-446.

[21] Hartl, R., Sethi, S.P. and R.G. Vickson (1995). A survey of the maximum principle for optimal control problems with state constraints. SIAM Review, 37, 181-218.

[22] Hindriks J, Peralta S. and Sh. Weber (2008). Competing in taxes and investment under fiscal equalization, Journal of Public Economics, Volume 92, Issue 12, Pages 2392-2402.

[23] Hu Y. and Mino K. (2014). Capital accumulation and structural change in a small open economy, Pacific Economic Review, Vol.19, 634-656.

[24] Itaya, J., Okamura, M., and C. Yamaguchi (2008). Are regional asymmetries detrimental to tax coordination in a repeated game setting? Journal of Public Economics 92, 2403-2411.

[25] Justman, M., J. F. Thisse and T. van Ypersele (2005). Fiscal competition and regional differentiation. Regional Science and Urban Economics, Vol. 35, Issue 6, 848-861.

[26] Kiyotaki N and J. Moore (1997). Credit cycles. Journal of Political Economy, 105(2), 211-248.

[27] Krauskopf B., H. Osinga, E. Doedel, M. Henderson, J. Guckenheimere, A. Vladimirsky, M. Dellnitz and O. Junge (2005). A survey of methods for computing (un)stable manifolds of vector fields. International Journal of Bifurcation and Chaos, 15, 763-792.

[28] La Porta, R., López-de-Silanes, F., Shleifer, A., Vishny, R. (1997). Trust in large organizations. American Economic Review Papers and Proceedings 97 (2), 333-339.

[29] Lorenz E. (1963). Deterministic nonperiodic flow. Journal of the Atmospheric Science 20, 130141.

[30] Mansoorian A. , G.M. Myers (1993). Attachment to home and efficient purchases of population in a fiscal externality economy. Journal of Public Economics $52,117-132$.

[31] Ogura L.M. ,(2006). A note on tax competition, attachment to home, and underprovision of public goods. Journal of Urban Economics 59, 252-258. 
[32] Pieretti P. and S. Zanaj (2011). On tax competition, Public Goods Provision and Jurisdictions' Size. Journal of International Economics, vol. 84(1), 124-130.

[33] Read R. (2008). Foreign Direct Investment in Small Island Developing States. Journal of International Development, 20, 502-525

[34] Streeten P. (1993). The Special Problems of Small Countries. World Development, 21(2), 197-202.

[35] Vaillancourt, François (1989). The administrative and compliance costs of the personal income tax and payroll tax system in Canada. Canadian Tax Papers, No. 86. Canadian Tax Foundation, c1989.

[36] Zissimos B. and M. Wooders (2008). Public good differentiation and the intensity of tax competition, Journal of Public Economics 92, 1105-1121. 